\title{
Selfsimilar Domain Growth, Localized Structures and Labyrinthine Patterns in Vectorial Kerr Resonators
}

\author{
R. Gallego, M. San Miguel and R. Toral \\ Instituto Mediterráneo de Estudios Avanzados, IMEDEA日 (CSIC-UIB), \\ Campus Universitat Illes Balears, E-07071 Palma de Mallorca, Spain.
}

(January 22, 2018)

\begin{abstract}
We study domain growth in a nonlinear optical system useful to explore different scenarios that might occur in systems which do not relax to thermodynamic equilibrium. Domains correspond to equivalent states of different circular polarization of light. We describe three dynamical regimes: a coarsening regime in which dynamical scaling holds with a growth law dictated by curvature effects, a regime in which localized structures form, and a regime in which polarization domain walls are modulationally unstable and the system freezes in a labyrinthine pattern.
\end{abstract}

Typeset using REVTEX 
The problem of the growth of spatial domains of different phases has been thoroughly studied in the context of the dynamics of phase transitions: a system is placed in an unstable state and one considers its relaxation to the state of thermodynamic equilibrium [1]. This process is dominated by the motion of domain walls and other defects. It is in this context that seminal ideas of selfsimilar evolution and dynamical scaling were introduced for nonequilibrium processes. Asymptotic domain growth laws, with their underlying physical mechanisms, have been well established, and dynamical scaling has been generally demonstrated. A growth law $R(t) \sim t^{1 / 2}$ holds for dynamics with no conservation law and domains made of equivalent phases. This law follows from the minimization of surface energy, and it has been shown to be robust against the appearance of point defects in systems with a discrete number of phases, three dimensional vortices or chiral domain walls [2]. Other well known growth laws [] are $R(t) \sim t^{1 / 3}$ for systems with conserved order parameter and $R(t) \sim t$ for nonconserved dynamics with a metastable phase [3], and also for hydrodynamic systems in spatial dimension $d>2$ [4].

Domain growth in systems that do not approach a final state of thermodynamic equilibrium is much less understood. For example, the mechanisms underlying a growth law $R(t) \sim t^{1 / 5}$ in pattern forming systems in which the spatial coupling is non purely diffusive (Swift-Hohenberg equation) [5] have not been clearly identified. Other general issues that need to be considered are the role of hamiltonian vs. dissipative dynamics [6], the effects of nonrelaxational dynamics such as one-dimensional motion of fronts between equivalent states and spiral formation [7], the emergence of localized structures (LS) [8, 9], or transverse instabilities of domain walls leading to labyrinthine patterns [10].

Driven nonlinear optical systems offer a wealth of opportunities for the study of pattern formation and other nonequilibrium processes in which the spatial coupling is caused by diffraction instead of diffusion. These systems are specially interesting because they naturally lead to the consideration of vectorial complex fields, being the vector character associated with the polarization of light, and also because they often support the formation of LS [11 [13]. Such bright light spots are being actively considered for applications in parallel 
optical processing. Only very recently domain growth has been considered in some of these systems and some growth laws obtained from numerical simulations have been reported [13 [15]. However, clear mechanisms for the growth laws have often not been identified, and some of these laws do not correspond unambiguously to an asymptotic regime. In addition, the question of dynamical scaling has, in general, not been addressed.

In this letter we consider a Kerr medium as a clear example of a nonlinear optical system in which many of the issues and scenarios mentioned above can be explored. We show that after switching-on a pump field, domain walls are formed which separate regions with different polarization of light. The dynamical evolution of these polarization domain walls leads to three different regimes. For high pump values there is a coarsening regime for which we demonstrate dynamical scaling with a growth law $R(t) \sim t^{1 / 2}$. For lower pump values this process is contaminated by the emergence of LS formed by the collapse of polarization domain walls to a stable bound structure. In a third regime the system evolves into a nearly frozen labyrinthine pattern caused by a transverse modulational instability of the polarization domain wall. These three qualitatively different regimes have been experimentally observed in another optical system [12] and considered in the realm of Swift-Hohenberg models [16].

Our calculations are based on a mean field model that describes the transverse spatiotemporal evolution of the two circularly polarized components of the electric field complex envelope, $E_{+}$and $E_{-}$, in an optical cavity filled with an isotropic self-defocusing Kerr medium and pumped with a linearly polarized real field $E_{0}$ [17,18]:

$$
\partial_{t} E_{ \pm}=-(1-i \theta) E_{ \pm}+i \nabla_{\perp}^{2} E_{ \pm}+E_{0}-\frac{1}{4} i\left[\left|E_{ \pm}\right|^{2}+\beta\left|E_{\mp}\right|^{2}\right] E_{ \pm} .
$$

Here $\theta$ is the cavity detuning, and $\nabla_{\perp}^{2}$ is the laplacian in the transverse plane. Equations (1) are damped and driven coupled Nonlinear Schrödinger equations which can be rewritten as

$$
\partial_{t} E_{ \pm}=-E_{ \pm}-i \frac{\delta \mathcal{F}}{\delta E_{ \pm}^{*}}
$$


where $\mathcal{F}\left[E_{+}, E_{-}\right]$is a real functional. Therefore, except for the linear dissipative term, the dynamics can be written in Hamiltonian form. This corresponds to a rather different dynamics than the normal relaxational dynamics considered in systems that approach a state of thermodynamic equilibrium. We will study different regimes for different values of the pump $E_{0}$.

Equations (11) admit symmetric $\left(I_{s+}=I_{s-}\right)$ and asymmetric $\left(I_{s+} \neq I_{s-}\right)$ steady state homogeneous solutions, where $I_{ \pm}=\left|E_{ \pm}\right|^{2}$. The homogeneous symmetric solution is linearly stable for $E_{0}<E_{0, a}$, while the asymmetric solutions only exist for $E_{0, a}<E_{0, b}<E_{0}$ and they are linearly stable for $E_{0, b}<E_{0, c}<E_{0}$ [18,19. There are two equivalent homogeneous stable solutions for $E_{0, c}<E_{0}$, one in which $I_{s+} \gg I_{s-}$ and the other one, obtained interchanging $E_{+}$ by $E_{-}$, in which $I_{s+} \ll I_{s-}$. These solutions are elliptically polarized, but very close to being circularly polarized, because one of the two circularly polarized components dominates. For simplicity we will call them the right and left circularly polarized solutions. If the pump field $E_{0}$ is switched-on from $E_{0}=0$ to a value $E_{0}>E_{0, c}$, only the mode with zero wavenumber can initially grow from the initial condition $E_{ \pm}=0$. One then expects that either of the two equivalent homogeneous solutions will locally grow and that domains separated by polarization walls will emerge. This is indeed the process that we study. We note, however, that a solution with a stripe pattern orthogonally polarized to the pump exists for $E_{0}>E_{0, a}[17,18$. This pattern solution is the one obtained by continuity from the homogeneous symmetric solution through a Turing-like instability. We have numerically checked that such solution remains stable for pump values $E_{0} \gg E_{0, c}$, but it is not the solution approached by the physical process just described of switching-on the pump to a value $E_{0}>E_{0, c}$.

We find three different dynamical regimes for $E_{0}>E_{0, c}$, summarized in fig. 11. For $E_{0}>E_{0,2}$ domains grow and the system coarsens, for $E_{0,2}>E_{0}>E_{0,1}$ stable LS are formed, while for $E_{0,1}>E_{0}>E_{0, c}$ a labyrinthine pattern emerges. These regimes are better understood by considering the evolution of an initial isolated polarization droplet: a circular domain of one of the solutions surrounded by the other solution. We find that the radius of 
the circular domain varies consistently with a curvature driven front motion. The normal front velocity $v_{n}$ (eikonal equation) follows a law of the form $v_{n}(\mathbf{r}, t)=-\gamma\left(E_{0}\right) \kappa(\mathbf{r}, t)$, where $\kappa$ is the local curvature of the domain wall and $\gamma\left(E_{0}\right)$ is a coefficient that depends on the pump field amplitude. For a circular domain we get $d R(t) / d t=-\gamma\left(E_{0}\right) / R(t)$. In figure 1 we show the function $\gamma\left(E_{0}\right)$ as obtained from the numerical solution of eqs. (1) in a twodimensional system for relatively large initial droplets. Notice that $\gamma\left(E_{0}\right)$ changes sign at $E_{0}=E_{0,1}$, which indicates a change from droplet shrinkage to droplet growth.

We first consider the regime of domain coarsening which occurs for $E_{0}>E_{0,2}$. In this regime $\gamma\left(E_{0}\right)>0$ and an isolated drop shrinks to zero radius. In the general dynamics starting from random initial conditions around $E_{ \pm}=0$, sharp domain walls are initially formed and they evolve reducing their curvature. The system approaches a final homogeneous state in which one of the two circularly polarized solutions fills the whole system. In order to characterize the coarsening process we have calculated the pair correlation function of $I_{+}$ and $I_{-}$, defined as $C_{I_{ \pm}}(\mathbf{r}, t)=\left\langle I_{ \pm}(\mathbf{x}+\mathbf{r}, t) I_{ \pm}(\mathbf{x}, t)\right\rangle$. The average $\langle\ldots\rangle$ is performed over the set of points $\mathbf{x}$ (and additionally over a set of 100 different random initial conditions). Due to the symmetry of the problem $C_{I_{+}}=C_{I_{-}} \equiv C$. Results for the circularly averaged correlation function $C(r, t)$ are shown in fig. 2. The mean size $L(t)$ of the domains is calculated as the distance at which $C(r, t)$ takes half its value at the origin, i.e., $C(L(t), t)=\frac{1}{2} C(0, t)$. We obtain a well defined asymptotic growth law $L(t) \sim t^{1 / 2}$ that follows from domain wall motion driven by curvature effects. We have further obtained that the dynamics is selfsimilar, i.e., that there is dynamical scaling. This is seen in fig. 2 where we plot $C(r, t)$ before and after rescaling the spatial coordinate of the system with the characteristic domain size $L(t)$. We observe that curves for different times in the scaling regime collapse to the single scaling function after rescaling. These results coincide with those obtained for many thermodynamic systems with nonconserved order parameter [1,2]. We note, however, that in our case the dynamics does not follow the minimization of any obvious energy and that surface tension is not a proper concept for the diffractive spatial coupling considered in optical systems. 
We next address the regime of formation of $\operatorname{LS}\left(E_{0,2}>E_{0}>E_{0,1}\right)$. In this regime, as in the previous case, $\gamma\left(E_{0}\right)>0$, and a large isolated droplet initially shrinks with a radius decreasing as $R(t) \sim t^{-1 / 2}$. However the shrinkage stops at a well defined final value of the radius. Initial droplets with a smaller radius grow to this final stable radius. In the general dynamics following the switch-on of the pump, domain walls are initially formed. They first evolve reducing their length as in the coarsening regime. But while in that regime a closed loop disappears, here it collapses to a stable LS formed by a bound state of the domain wall. The final state is composed of stretched domain walls and LS. To understand this process is convenient to consider the form of the polarization domain walls in a $d=1$ geometry, as shown in figure 3. An isolated $d=1$ domain wall is stationary. We observe that the intensity profiles of the walls do not approach monotonically the asymptotic value of the homogeneous state. When several domain walls are created in the transient dynamics, they interact with each other. Since the front profiles have oscillatory tails, the interaction between two walls can lead to repulsive forces [8]. As a consequence, LS formed by bound domain walls can be formed which stop the coarsening process. These oscillatory tails are less important the larger is $E_{0}$ (see figure [3). However, we find that for all the values of $E_{0}$ which we have explored (up to $E_{0}=10$ ), this effect is enough to stop coarsening in $d=1$ : a frozen pattern state is always dynamically reached [20]. What happens in our $d=2$ situation is a competition between the $d=1$ repulsive effect between walls and the curvature effect that tends to reduce a droplet to zero radius. When the repulsive force is large enough, it might counterbalance the shrinkage process driven by curvature, and thus leads to the formation of a LS. This happens for $E_{0,1}<E_{0}<E_{0,2}$. The mechanism is the one also discussed in [13]. These structures can be seen as a hole of $I_{+}\left(I_{-}\right)$in the background of a circularly +- polarized $(--$ polarized $)$ state, together with a peak of $I_{-}\left(I_{+}\right)$. Since the oscillatory tails are larger as $E_{0}$ decreases, the size of the LS decreases with $E_{0}$. We have

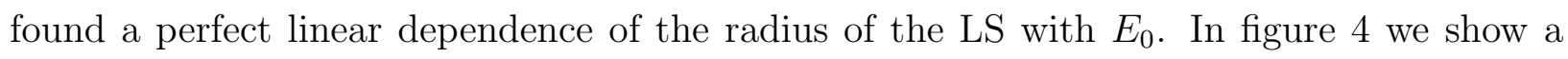
plot of a LS together with its transverse profile. Note that the intensity in the LS is greater than in the surrounding background. 
We finally discuss the regime of labyrinthine pattern formation which occurs for $E_{0}<$ $E_{0,1}$ : switching-on the pump produces a very dense pattern of domain walls that repel each other. In this regime $\gamma\left(E_{0}\right)<0$, and an isolated droplet of arbitrary small size grows as $R(t) \sim t^{1 / 2}$. In an infinitely large system the droplet would grow without limit, but with periodic boundary conditions it grows until the domain wall interacts with itself. Repulsion of the domain wall leads to a labyrinthine pattern as shown in fig. 5. An independent way of identifying the value $E_{0}=E_{0,1}$, below which labyrinthine patterns emerge, is by a linear stability analysis in $d=2$ of the $d=1$ domain wall profile. We have numerically obtained that such flat domain wall has a transverse modulational instability for values of the pump amplitude for which $\gamma\left(E_{0}\right)<0$. We find a longwavelength instability in which arbitrary small wavenumbers become unstable for $E_{0}<E_{0,1}$ (see fig. 6). This is reminiscent of the situation described for vectorial Second Harmonic Generation [15]. In physical terms, both the droplet growth and the modulational instability indicate that the system prefers to have the longest possible domain walls, or equivalently the largest possible curvature. This leads to a nearly frozen state in which the oscillatory tails of the domain walls prevent their selfcrossing and in which coarsening is suppressed. LS might form, but their natural tendency to grow is stopped by surrounding walls.

In summary, we have described a situation in nonlinear optics in which many of the generic issues and possible scenarios of domain growth in nonthermodynamic systems occur. In spite of the nonrelaxational dynamics we have found a regime of selfsimilar evolution with a growth law characteristic of curvature driven motion. In other regimes, obtained just by changing the pump amplitude, domain growth is contaminated by the emergence of LS or suppressed by an instability of the domain wall that leads to a nearly frozen labyrinthine pattern. Domain walls and LS are here associated with the polarization vectorial degree of freedom of light.

Financial support from DGICYT (Spain, Projects PB94-1167 and PB97-0141-C02-01) is acknowledged. Helpful discussions with P. Colet, M. Hoyuelos and B. Malomed are also acknowledged. 


\section{REFERENCES}

* Electronic address: http://www.imedea.uib.es/PhysDept/

[1] J.D. Gunton, M. San Miguel and P. Sahni in Phase Transitions and Critical Phenomena, vol. 8, pp. 269-466, edited by C. Domb and J. Lebowitz (Academic Pres, 1983); M. San Miguel, A. Amengual, E. Hernández-García, Phase Transitions 48, 65 (1994).

[2] K. Kaski et al., Phys. Rev. B 31, 3040 (1985); M. Mondello and N. Goldenfeld, Phys. Rev. A 45,657 (1992); H. Tutu, Phys. Rev. E 56, 5036 (1997).

[3] I.M. Lifshitz, Sov. Phys. JETP (1962).

[4] M. San Miguel, M. Grant and J.D. Gunton, Phys. Rev. A 31, 1001 (1985).

[5] M.C. Cross and D.I. Meiron, Phys. Rev. Lett. 75, 2152 (1995).

[6] C. Josserand and S. Rica, Phys. Rev. Lett. 69, 1215 (1997).

[7] R. Gallego, M. San Miguel, and R. Toral, Phys. Rev. E 58, 3125 (1998); Physica A 257, 207 (1998).

[8] P. Coullet, C. Elphick, and D. Repaux, Phys. Rev. Lett. 58, 431 (1987).

[9] T. Ohta, Physica D 34, 115 (1989); D. Battogtokh, M. Hildebrand, K. Krischer, and A.S. Mikhailov, Phys. Reports 288, 435 (1997); H. Riecke, in Pattern formation in continuous and coupled systems, edited by M. Golubitsky, D. Luss and S.H. Strogatz (Springer, New York, 1999)

[10] R.E. Goldstein, D.J. Muraki, and D.M. Petrich, Phys. Rev. E 53, 3933 (1996).

[11] M. Tlidi, P. Mandel and R. Lefever, Phys. Rev. Lett. 73, 640 (1994); W. Firth and A.J. Scroggie, Phys. Rev. Lett. 76, 1623 (1996); C. Etrich, U. Peschel, and F. Lederer, Phys. Rev. Lett. 79, 2454 (1997); M. Brambilla et al., Phys. Rev. Lett. 79, 2042 (1997); K. Staliunas and J.V. Sánchez-Morcillo, Phys. Rev. A 57, 1454 (1998). 
[12] V.B. Taranenko, K. Staliunas, and C.O. Weiss, Phys. Rev. Lett. 81, 2236 (1998).

[13] G.L. Oppo, A.J. Scroggie, and W.J. Firth, Quantum Semiclass. Opt. 1, 133 (1999) [These authors have also considered dynamical scaling in an Optical Parametric Oscillator].

[14] M. Tlidi, P. Mandel and R. Lefever, Phys. Rev. Lett. 81, 979 (1998); M. Tlidi and P. Mandel, Europhys. Lett. 44, 449 (1998).

[15] U. Peschel, D. Michaelis, C. Etrich and F. Lederer, Phys. Rev. E 58, R2745 (1998).

[16] K. Ouchi and H. Fujisaka, Phys. Rev. E 54, R3895 (1996).

[17] J.B. Geddes, J.V. Moloney, E.M. Wright and W.J. Firth, Opt. Comm. 111, 623 (1994).

[18] M. Hoyuelos, P. Colet, M. San Miguel, and D. Walgraef, Phys. Rev. E 58, 2292 (1998).

[19] For the parameter values used throughout this paper, $\theta=1$ and $\beta=7$ [18], one finds $E_{0, a}=0.89, E_{0, b}=1.02, E_{0, c}=1.14$.

[20] A final homogeneous state is obtained when the pump is not linearly polarized $\left(E_{0,+} \neq\right.$ $\left.E_{0,-}\right)$. An isolated domain wall is then no longer stationary. 


\section{FIGURES}

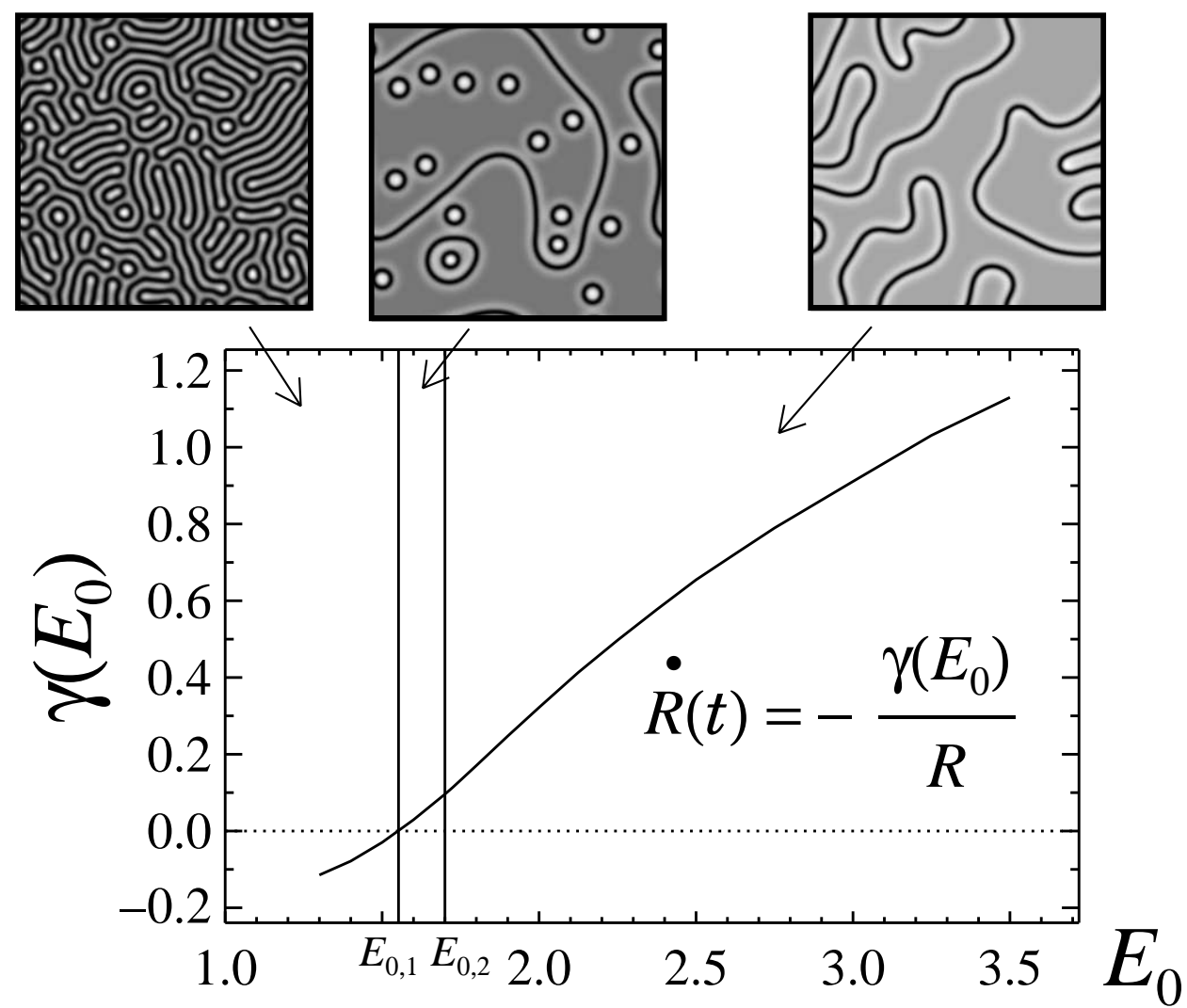

FIG. 1. Coefficient $\gamma\left(E_{0}\right)$ as defined in the text. Snapshots of typical configurations for the total intensity $I=I_{+}+I_{-}$at late times are shown for each of the three dynamical regimes. The vertical lines identify the values of $E_{0,1}=1.552$ and $E_{0,2}=1.700$. 


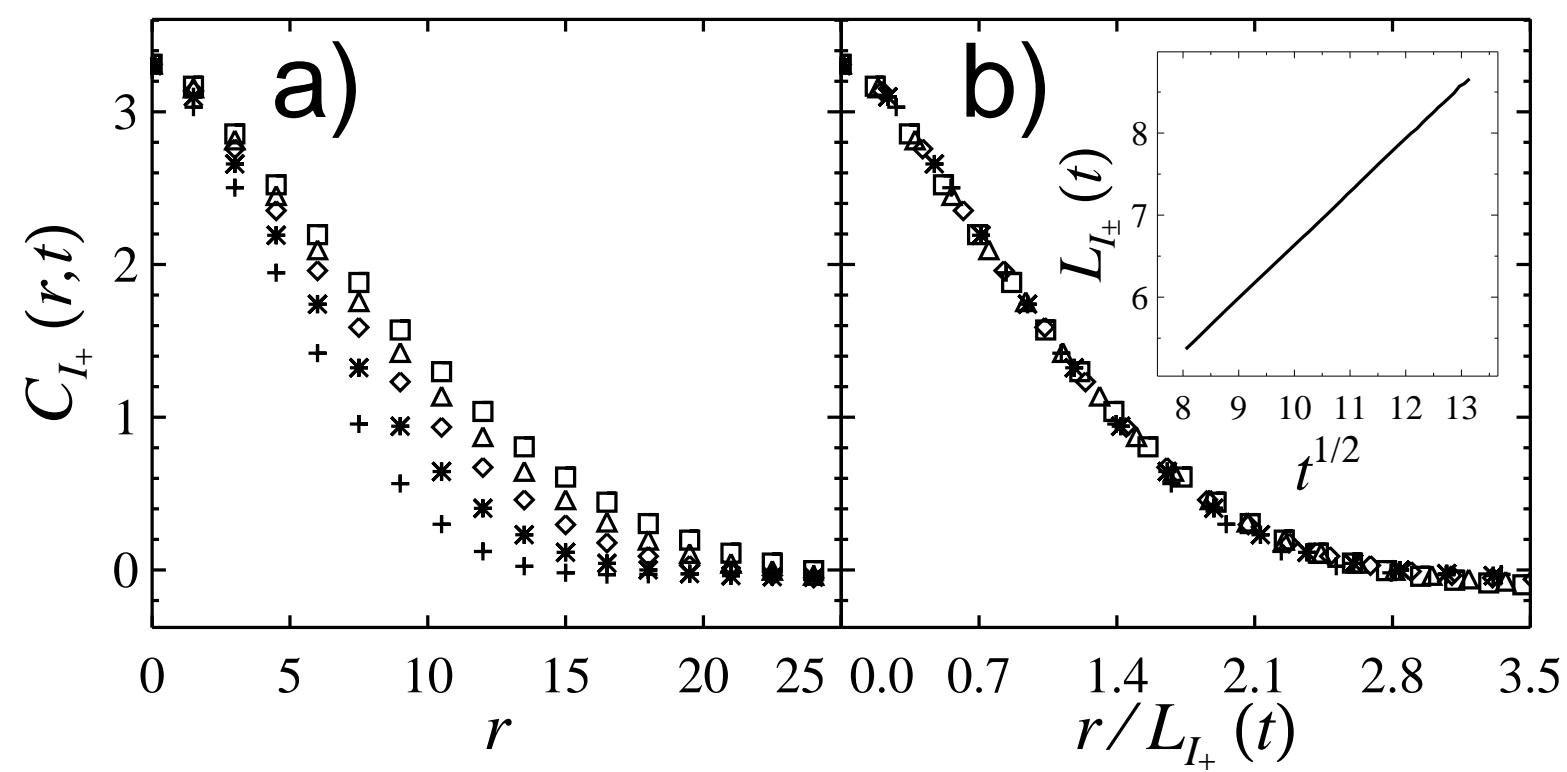

FIG. 2. a) Spherical averaged correlation function for $E_{0}=1.8>E_{0,2}$ at times $t=64.8,91.8$, 11.8, 145.8 and 172.8 and b) after scaling $r$ with the domain size $L_{I_{+}}(t)$. The inset shows the domain growth law $L_{I_{+}}(t) \sim t^{1 / 2}$.

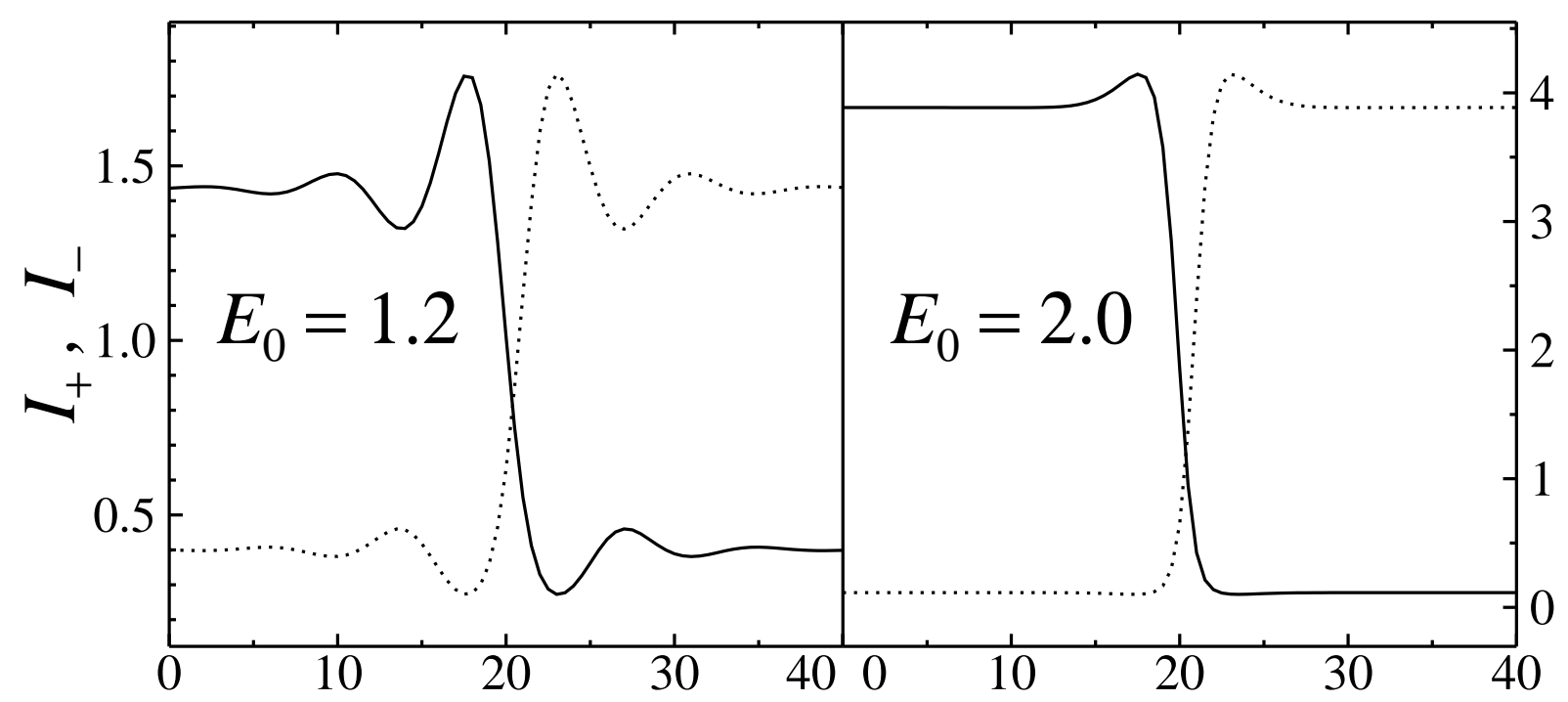

FIG. 3. $d=1$ intensity profiles of the polarization domain wall for two pump values: $E_{0}=1.2<E_{0,1}, E_{0}=2>E_{0,2}$. 


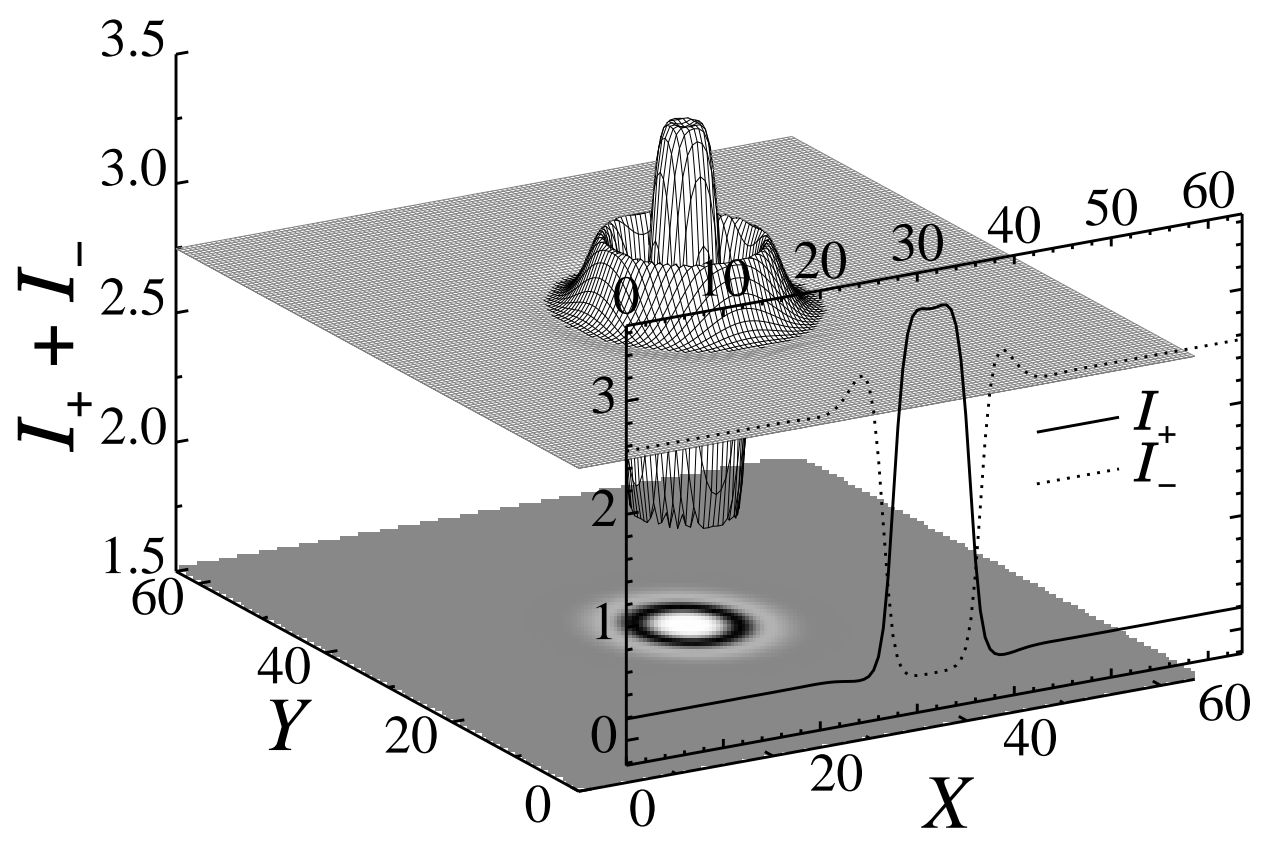

FIG. 4. Total field intensity $\left(I_{+}+I_{-}\right)$of a LS and transverse profile of $I_{+}$and $I_{-}$for $E_{0}=1.6$.
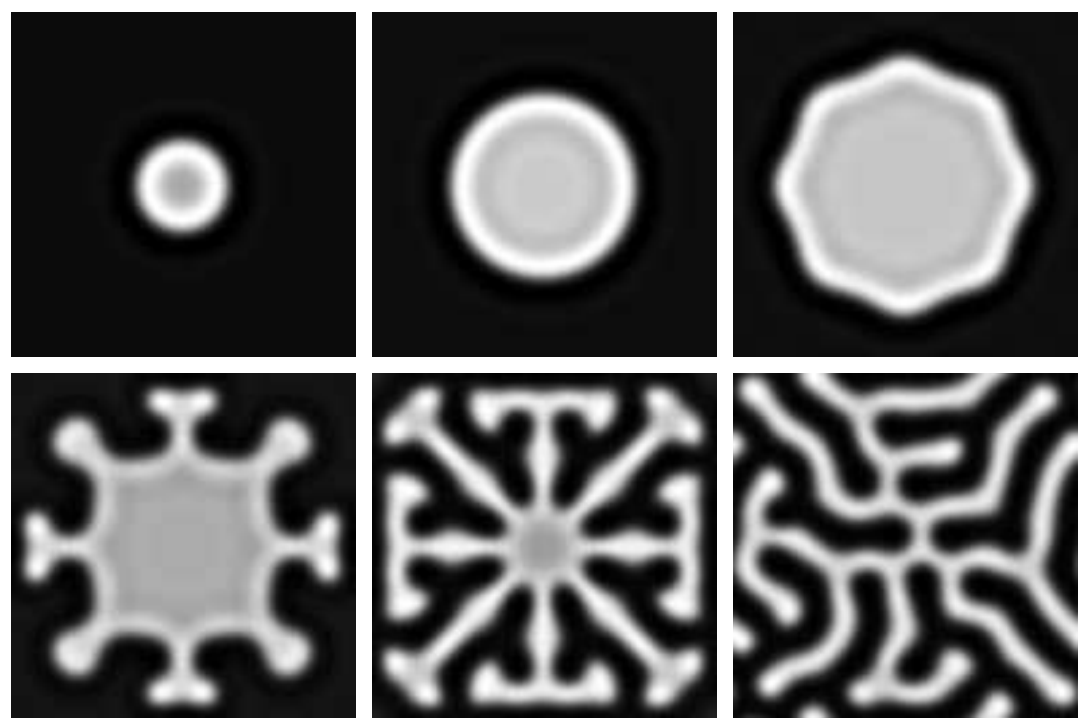

FIG. 5. Growth of a polarization droplet and creation of a labyrinthine pattern for $E_{0}=1.3<E_{0,1}$. Snapshots at times $t=0,1000,2100,2400,3200$ and 4900. 


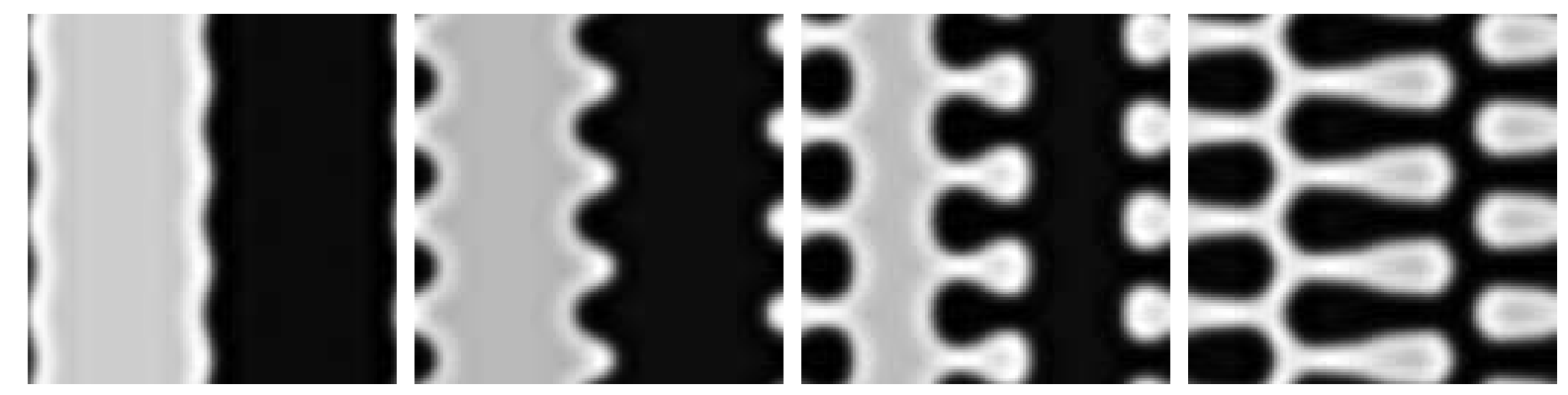

FIG. 6. Transverse modulational instability for a flat domain wall. $E_{0}=1.4$. Snapshots at times $t=0,400,600,860$. 